WILEY-VCH

\title{
Optical Antennas for Wavelength Division Multiplexing in Visible Light Communications beyond the Étendue limit
}

Pavlos P. Manousiadis, Hyunchae Chun, Sujan Rajbhandari, Dimali A. Vithanage, Rahmat Mulyawan, Grahame Faulkner, Harald Haas, Dominic C. O'Brien, Steve Collins, Graham A. Turnbull*, and Ifor D.W. Samuel*

Dr Pavlos P. Manousiadis, Dr Dimali A. Vithanage, Prof. Graham A. Turnbull, and Prof. Ifor D.W. Samuel

Organic Semiconductor Centre, SUPA, School of Physics and Astronomy, University of St Andrews, St. Andrews, KY16 9SS, United Kingdom.

E-mail: i. G. A. Turnbull: gat@st-andrews.ac.uk, ii. I. D. W. Samuel: idws@st-andrews.ac.uk

Dr Hyunchae Chun, Dr Sujan Rajbhandari, Mr Rahmat Mulyawan, Mr Grahame Faulkner, Prof. Dominic C. O’Brien, Prof. Steve Collins

Department of Engineering Science, University of Oxford, Oxford, OX1 3PJ, United Kingdom.

Dr Hyunchae Chun

Current address: Department of Information and Telecommunication Engineering, Incheon National University, Incheon, 22012, Korea.

Dr Sujan Rajbhandari

Current address: School of Computing, Electronics and Mathematics, Coventry University, Coventry, CV1 2JH, United Kingdom.

Prof. Harald Haas

Li-Fi Research and Development Centre, Institute for Digital Communications, University of Edinburgh, Edinburgh EH8 9YL, United Kingdom.

Keywords: optical wireless communications, fluorescent concentrator, Li-Fi, field-of-view.

\section{Abstract}

This paper presents a novel fluorescent receiver for Visible Light Communications that combines a broad field-of-view with a significant gain, and simultaneously enables wavelength-division-multiplexing for data communications. These characteristics are achieved by creating a receiver that includes a stack of two fluorescent optical antennas, each designed to receive a different part of the spectrum and create two separable communication channels. The device outperforms the theoretical étendue limit in terms of the combination of field-of-view and gain it can achieve. Furthermore, the demonstrated de-multiplexing of blue and green spectral components enables the parallel streaming of data by wavelength division 


\section{WILEY-VCH}

multiplexing. Since these devices are inexpensive, simple and compact, they can easily be integrated into portable electronic devices such as phones, tablets, and laptops.

\section{Introduction}

Visible light communication (VLC) is a new paradigm for wireless communications that has the potential to supplement conventional radio-frequency (RF) based wireless communication methods. ${ }^{[1,2]}$ Similar to all wireless communication, there is a need for antennas to increase the signal-to-noise ratio (SNR) at the receiver end of the data link to enable or enhance data transfer. In VLC receivers, SNR is typically increased by an optical element, most commonly a lens or a compound parabolic concentrator ${ }^{[3,4]}$ However, the use of these optical elements restricts the field-of-view (FoV), meaning that VLC links are more sensitive to the alignment of the transmitter and the receiver than RF links. This sensitivity is due to a fundamental principle in optics, the conservation of étendue, which creates a trade-off between the gain, and thus the increase in SNR provided by the optical system, and the FoV of the system, ${ }^{[5]}$ as Figure 1a and $\mathbf{b}$ illustrate. This presents a challenge for implementing a practical VLC link for mobile applications e.g. to a laptop or smartphone, because of the need for constant careful alignment. ${ }^{[4]}$

A recent series of publications have demonstrated a novel optical solution to the constraints imposed by étendue using a fluorescent antenna. ${ }^{[6,7]}$ It was demonstrated that the gain of the fluorescent optical antenna outperformed by three times the theoretical limit for any refraction-reflection based optical system with the same FoV ${ }^{[6]}$ The structure consisted of a fluorescent material sandwiched between two glass layers, as shown in Figure 1c. Due to the higher refractive index of the fluorescent layer, part of the fluorescence is trapped by total internal reflection. As a result, this light is guided through the film and escapes at the edges. The relatively large surface area for absorbing the incoming signal, compared with small edge 


\section{WILEY-VCH}

area of emission leads to an enhancement of the signal's power density, increasing the SNR of the system.

In addition to their other advantages, the selective absorption spectra of fluorophores offers

the opportunity to use wavelength-division multiplexing (WDM) to increase link capacity. ${ }^{[1,8]}$ In order to construct a reliable WDM link, the key challenge is to separate, at the receiver side, the individual data streams carried by different wavelengths whilst minimising link loss and crosstalk between these data streams. Applying absorbing or reflecting optical colour filters in front of individual photo-detectors is the most popular approach to $\mathrm{WDM}^{[9,10]}$. For example, Wu et al. ${ }^{[10]}$ reported a WDM system, which uses colour selective dichroic filters to separate individual WDM links. This structure makes all colour channels propagate along the same optical axis, maintaining collinearity, and compact design, but supports only point-topoint applications with limited FoV.

In this letter, we demonstrate a simple, inexpensive and practical device, based on fluorescent optical antennas, which can enable WDM in VLC and thus enable a further improvement in link capacity. The concept of this device is illustrated in Figure 2. The device is a stack of two fluorescent optical antennas each designed to preferentially absorb a particular part of the visible light spectrum and therefore concentrate a separate colour channel. The results show that this fluorescent antenna can enhance data rates, whilst also achieving good gain and FoV.

\section{Device fabrication and characterization}

The requirements for the emitting material in a fluorescent antenna are ${ }^{[11]}$ : (i) strong absorption in the emission wavelength; (ii) high fluorescence quantum yield (PLQY); (iii) excited states with a lifetime of less than $10 \mathrm{~ns}$ for $\mathrm{MHz}$ range bandwidth, preferably closer to $1 \mathrm{~ns}$; and (iv) weak overlap between absorption and emission spectra. Further challenges for 


\section{WILEY-VCH}

materials and fabrication of a WDM enabling fluorescent antenna are: (v) a selective colour absorption of the incident light, and (vi) an effective separation and extraction of multiple wavelengths.

The two dyes which fulfil the requirements for the materials for de-multiplexing the blue and green components of the light signal are: Coumarin 6 (Cm6) and 4-(Dicyanomethylene)-2methyl-6-(4-dimethylaminostyryl)-4H-pyran (DCM), respectively. Each material was incorporated into an antenna composed of a fluorescent layer, where the dye molecules were dispersed in a transparent epoxy matrix, sandwiched between two microscope slides. The two antennas were then stacked to make the WDM antenna. Figure 3, shows the basic results of the photophysics study of the two materials, with absorption spectra of the layers shown in Figure S3. Cm6 layer has a strong absorption in the region of the blue LED (optical density (OD) 2.25 at $465 \mathrm{~nm}$ ), while transmitting most of the green part of the spectrum (OD below 0.1 beyond $510 \mathrm{~nm}$ ). The absorption of the DCM layer extends into the region of the green LED's emission (OD 1.3 at $525 \mathrm{~nm}$ ). The emission spectra do not strongly overlap, with Cm6 having an emission peak at $510 \mathrm{~nm}$ and DCM at $610 \mathrm{~nm}$. Cm6 has a high PLQY of 80-95\%, while DCM has a PLQY of $50 \%$. Both dyes show a single exponential PL decay with a similar lifetime of $\leq 3 \mathrm{~ns}$ (see Figure 3c; $2.57 \mathrm{~ns}$ for $\mathrm{Cm} 6,2.70 \mathrm{~ns}$ for DCM).

The WDM data link consisted of the WDM antenna placed under an optical transmitter, with an optical detector at either end, as shown in Figure 2. The transmitter was a commercial multi-chip RGB LED. The blue $(\lambda \max \approx 460 \mathrm{~nm}, \mathrm{FWHM} 23 \mathrm{~nm})$ and the green $(\lambda \max \approx 520$ nm, FWHM $36 \mathrm{~nm}$ ) LED chips were driven by an arbitrary waveform generator, applying independent data-streams to the two sources. The intensities of both LEDs were adjusted so that the incident light on the WDM antenna from each LED was $1 \mathrm{~W} / \mathrm{m} 2$, with calculated lux to be 111 lux for the blue LED, and 665 lux for the green LED. For comparison, the standard 


\section{WILEY-VCH}

for a good illuminated office environment is 500 lux of white light (CIBSE recommended lighting levels), which corresponds to about $5 \mathrm{~W} / \mathrm{m} 2$ of white light from a typical blue coated blue LED.

The WDM antenna was placed $20 \mathrm{~cm}$ from the LED sources, with the $\mathrm{Cm} 6$ layer closer to the sources. Due to the high absorption of the blue part of the spectrum $(450-470 \mathrm{~nm})$ by Cm6, only a small portion of the blue light reached the underlying DCM layer. On the other hand, Cm6 has low absorption of green light $(\sim 520 \mathrm{~nm})$, therefore most of the green light was transmitted to the layer of DCM where it was absorbed. Finally, the output from each of the fluorescent antennas was butt-coupled to a photodiode (see Figure 2).

The advantage of the fluorescent antenna over the traditional optics is the combination of the wide FoV and gain that it can achieve. Figure 4 summarises the measurements of gain of the two antennas as a function of incident angle. For the Cm6 layer, the gain measured using the blue LED, is approximately 8 , while for the DCM layer, the gain from the green LED, is approximately 11. In both cases, we can see from this figure that the FoV is much larger than the FoV of a condenser lens which would give a similar optical gain.

\section{Communication performance}

To evaluate the de-multiplexing capabilities of the WDM fluorescent antenna, the cross-talk between the WDM channel was measured. Like in the case of spatial multiple-input-multipleoutput (MIMO) systems, the channels $H$ matrix shows the proportions of the intended signals and the crosstalk. This can be measured by transmitting known training sequences and recording the received signal on each photodiode. Equation (1) shows both the definition of the normalised channel $H$ matrix of the system and the measured values for the particular WDM fluorescent antenna that was tested: 


\section{WILEY-VCH}

$$
\left[\begin{array}{c}
S_{C m 6} \\
S_{D C M}
\end{array}\right]=\mathbf{H}\left[\begin{array}{c}
S_{\text {blue }} \\
S_{\text {green }}
\end{array}\right] ; \quad \mathbf{H}=\left[\begin{array}{cc}
0.91 & 0.36 \\
0.43 & 1
\end{array}\right] ;
$$

where $S_{C m 6}$ and $S_{D C M}$ are the received PL signals from Cm6 and DCM, and $S_{\text {blue }}$ and $S_{\text {green }}$ are the transmitted signals from blue and green LED, respectively. The $H$ matrix also indicated about the levels of fluorescent light measured by the photodiodes, for each LED. For the blue LED, about $68 \%$ is collected by the photodiode attached to the Cm6 doped layer and $32 \%$ by the photodiode attached to the DCM doped layer. For the green LED, these percentages are $26 \%$ and $74 \%$, respectively. In terms of cross talk, the $\mathrm{H}$ matrix indicates that for both layers the cross-talk is about $30 \%$.

The values in the $\mathbf{H}$ matrix show that crosstalk is less than half of the desired signal. To eliminate the residual inter-channel crosstalk and hence separate the channels, standard MIMO processing techniques can be used, the simplest of which is apply the inverse of $\mathbf{H}\left(\mathbf{H}^{-}\right.$ 1) at the receiver (also known as zero-forcing, ZF). In the absence of noise, the product of $\mathbf{H H}^{-1}$ is an identity matrix, hence the crosstalk is completely eliminated. Though the ZF enhances noise and other advanced techniques such as minimum mean square estimation (MMSE), iterative interference cancellation, or pre-coding at the transmitter exists, the performance difference of these techniques are minimal ${ }^{[12]}$. Hence, ZF was applied in this work due to its simplicity.

In order to test the link, using on-off keying (OOK) scheme a stream of $2^{14}$ pseudorandom bits was transmitted to the WDM antenna. Figure 5 shows the measured bit-error-rate (BER) as a function of the data rate for the two channels. Without channel separation, the different colour channels were indistinguishable, leading to high BERs for all data-rates (grey lines in Figure 5). When the WDM fluorescent antenna was used, a $1 \mathrm{Mb} / \mathrm{s}$ data rate with BER of $10^{-4}$ was achieved, simultaneously, for both blue and green channels. The superimposition of two OOK waveforms of different amplitude (one of these being due to the crosstalk from the unwanted 


\section{WILEY-VCH}

channel) can clearly be seen on the $1 \mathrm{Mb} / \mathrm{s}$ eye diagram. Despite this, a low BER link can be achieved with subsequent MIMO processing.

At higher data rates, the unprocessed eye diagrams become hard to discern due to the limited bandwidth of the photodiodes used (2 MHz). Nevertheless, after applying MIMO processing and (temporal) equalisation techniques, a good eye-opening is created at $6 \mathrm{Mb} / \mathrm{s}$, resulting in error-free operation. An aggregate, $12 \mathrm{Mb} / \mathrm{s}$ was therefore achieved by taking advantage of the colour separating characteristic of the WDM antenna.

Higher data rates would be achieved by using higher bandwidth photodiodes. However, this experiment shows that a compact WDM antenna can be used to substantially increase available data rate, whilst maintaining a large FoV.

\section{Conclusion}

In conclusion, this letter presents a novel, yet simple and compact device, which can combine a wide FoV, high gain, and enable WDM. The device is based on a stacked two-layered fluorescent optical antenna. It has been shown that the device outperforms traditional optics (which are limited by the conservation of étendue) giving significant signal gain, combined with a large FoV. In particular, we have achieved gains of more than 8 and 11, and a wide FoV, while enabling WDM. Finally, it was demonstrated experimentally that this device can enable practical WDM VLC receivers. This approach opens a new pathway to spectrally efficient, high gain receivers with a small form factor that could be integrated into mobile devices so that they have a VLC capability.

\section{Experimental Section}

Device fabrication. The dyes Coumarin 6 (Cm6) and 4-(Dicyanomethylene)-2-methyl-6-(4dimethylaminostyryl)-4H-pyran (DCM), where chosen for de-multiplexing the blue and green components of the light signal, respectively. The two components of the device were fabricated using the same method. Each dye was separately mixed with the UV curable 


\section{WILEY-VCH}

epoxy, Norland 68 (NOA68). For each solution, a concentration of $4 \mathrm{mg} / \mathrm{ml}$ was used. The solutions were prepared by weighing the appropriate mass of dye then adding the corresponding volume of epoxy. The solution was mixed, with a magnetic stirring bar for 24 hours. The ends of two microscope slides were covered in black tape that was trimmed to the same thickness as the microscope slide using a scalpel. A volume of ca. $0.25 \mathrm{ml}$ of dye/epoxy solution was spread on the slide which was placed between four spacers on a flat steel plate (see Figure S1). The spacers were made from aluminium, in a disk shape and their thickness was chosen to be the thickness of the two microscope slides plus $100 \mu \mathrm{m}$. A second microscope slide was placed on top of the first slide. These were pressed together under a uniform pressure from a $3 \mathrm{~mm}$ thick fused silica plate so that the thickness of the antenna was defined by the spacer thickness. The epoxy was then cured by a flood UV exposure, using an $8 \mathrm{~W}$ UV lamp at $365 \mathrm{~nm}$, for three minutes. The two components where attached to each other by an insulated tape (see Figure S2). Between the two components a small gap was formed ( $\sim 0.1 \mathrm{~mm})$ by using two pieces of tape as spacers.

Photophysical measurements. In order to avoid self-absorption of emission, low concentration films were used for phot physical studies. The maximum absorbance of $\mathrm{Cm} 6$ in NOA68 was 0.25 , while for DCM in NOA68 was 0.4. The absorbance of the films was measured with a Cary 300 (Varian Inc.) spectrophotometer. The photoluminescence (PL) spectra and lifetimes were measured with a FLS980 fluorimeter (Edinburgh Instruments Ltd.) with an excitation wavelength of $450 \mathrm{~nm}$. PL lifetime measurements were taken using the FLS980 with a PicoQuant laser, exciting at $375 \mathrm{~nm}$ to excite the sample and a photomultiplier tube (PMT) for detection. The photoluminescence quantum yield (PLQY) was measured with an integrating sphere $^{[13]}$ (Hamamatsu integrating sphere C9920-02 Luminescence measurement system) under excitation at $450 \mathrm{~nm}$.

Gain measurements. The gain for different channels was measured by placing the device on a rotating stage and comparing it to the incident power. The measurements for lens gain where 


\section{WILEY-VCH}

done by a condenser lens ACL50832U (Thorlabs), where the APD430A (Thorlabs) was used as a detector. The spot size was adjusted by positioning the lens at an appropriate distance from the detector, so that to achieve gain similar to that of the fluorescent concentrator. For illumination diode laser D405-120 was used (Digikey), with an engineered diffuser ED1S20(-MD) (Thorlabs).

Data communication measurement. The LEDs used were LZ4-00MC00 (LED Engine Inc.) with a bandwidth of $4 \mathrm{MHz}$. The S2387-130R (Hamamatsu) silicon photo-detectors, with an effective active area of $35 \mathrm{~mm}^{2}$ and bandwidth of $2 \mathrm{MHz}$, were aligned to the edges of the WDM fluorescent antenna (see Figure 2). An arbitrary waveform generator (Agilent 81150A) and real-time storage oscilloscope (Agilent MSO7104B) were used for the BER vs. data-rate measurement.

\section{Research data}

The research data supporting this publication can be accessed at http://dx.doi.org/10.17630/77059e57-6996-4500-95fe-143f56802e80.

\section{Supporting Information}

Supporting Information is available from the Wiley Online Library or from the author.

\section{Acknowledgements}

This research work was supported by the EPSRC via "Ultra-parallel visible light communications (UP-VLC)"(Programme Grant EP/K00042X/1) and "Super Receivers for Visible Light Communications" (related grants EP/R00689X/1 and EP/R005281/1).

Received: ((will be filled in by the editorial staff))

Revised: ((will be filled in by the editorial staff)) Published online: ((will be filled in by the editorial staff))

\section{References}

[1] H. Chun, S. Rajbhandari, G. Faulkner, D. Tsonev, E. Xie, J. J. D. McKendry, E. Gu, M. D. Dawson, D. O'Brien, H. Haas, Journal of Lightwave Technology 2016, 34, 3047.

[2] H. Chun, P. Manousiadis, S. Rajbhandari, D. A. Vithanage, G. Faulkner, D. Tsonev, J. J. D. McKendry, S. Videv, E. Xie, E. Gu, M. D. Dawson, H. Haas, G. A. Turnbull, I. D. W. Samuel, D. O'Brien, IEEE Photonics Technology Letters 2014, 26, 2035; H. Chun, A. Gomez, G. Faulkner, D. O’Brien, Opt. Lett. 2018, 43, 2708.

[3] J. M. Kahn, J. R. Barry, Proceedings of the IEEE 1997, 85, 265. 


\section{WILEY-VCH}

[4] D. Wang, T. Lan, Appl. Opt. 2018, 57, 1510.

[5] H. A. Hajjar, B. Fracasso, F. Lamarque, "Mini optical concentrator design for indoor high bit rate optical wireless communications", presented at Optical Wireless Communications (IWOW), 2013 2nd International Workshop on, 21-21 Oct. 2013, 2013; S. M. I. Roberto Ramirez-Iniguez, Ziran Sun, Optical Wireless Communications. IR for Wireless Connectivity, Auerbach Publications, 2008; X. Ning, R. Winston, J. O'Gallagher, Appl. Opt. 1987, 26, 300 .

[6] P. P. Manousiadis, S. Rajbhandari, R. Mulyawan, D. A. Vithanage, H. Chun, G. Faulkner, D. C. O’Brien, G. A. Turnbull, S. Collins, I. D. W. Samuel, Optica 2016, 3, 702. [7] T. Peyronel, K. J. Quirk, S. C. Wang, T. G. Tiecke, Optica 2016, 3, 787; Y. Dong, M. Shi, X. Yang, P. Zeng, J. Gong, S. Zheng, M. Zhang, R. Liang, Q. Ou, N. Chi, S. Zhang, Opt. Express 2017, 25, 21926.

[8] Y. Wang, L. Tao, X. Huang, J. Shi, N. Chi, IEEE Photonics Journal 2015, 7, 1; P. Manousiadis, H. Chun, S. Rajbhandari, R. Mulyawan, D. A. Vithanage, G. Faulkner, D. Tsonev, J. J. D. McKendry, M. Ijaz, E. Xie, E. Gu, M. D. Dawson, H. Haas, G. A. Turnbull, I. D. W. Samuel, D. O. Brien, "Demonstration of $2.3 \mathrm{~Gb} / \mathrm{s}$ RGB white-light VLC using polymer based colour-converters and GaN micro-LEDs", presented at 2015 IEEE Summer Topicals Meeting Series (SUM), 13-15 July 2015, 2015; G. Cossu, W. Ali, R. Corsini, E. Ciaramella, Opt. Express 2015, 23, 15700.

[9] Y. Wang, Y. Wang, N. Chi, J. Yu, H. Shang, Opt. Express 2013, 21, 1203.

[10] F. M. Wu, C. T. Lin, C. C. Wei, C. W. Chen, Z. Y. Chen, H. T. Huang, S. Chi, IEEE Photonics Journal 2013, 5, 7901507.

[11] E. Yablonovitch, J. Opt. Soc. Am. 1980, 70, 1362; G. Smestad, H. Ries, R. Winston, E. Yablonovitch, Sol. Energy Mater. 1990, 21, 99; J. S. Batchelder, A. H. Zewai, T. Cole, Appl. Opt. 1979, 18, 3090; J. S. Batchelder, A. H. Zewail, T. Cole, Appl. Opt. 1981, 20, 3733. [12] A. Burton, H. L. Minh, Z. Ghassemlooy, E. Bentley, C. Botella, IEEE Photonics Technology Letters 2014, 26, 945.

[13] N. C. Greenham, I. D. W. Samuel, G. R. Hayes, R. T. Phillips, Y. A. R. R. Kessener, S. C. Moratti, A. B. Holmes, R. H. Friend, Chem. Phys. Lett. 1995, 241, 89.
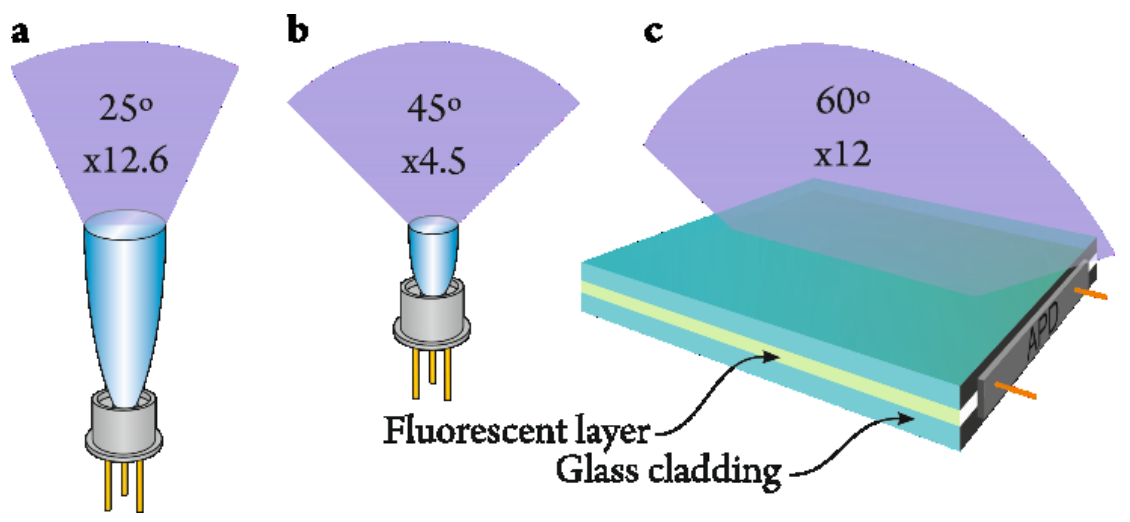

Figure 1. Examples of achievable fields-of-view and optical gain for different optical receivers. a) The maximum theoretical gain for a compound parabolic concentrator with a field-of-view of $25^{\circ}$. b) The maximum theoretical gain for a compound parabolic concentrator with a field-of-view of $45^{\circ}$. c) Experimental results for the field-of-view and gain of a fluorescent antenna ${ }^{[6]}$. 


\section{WILEY-VCH}

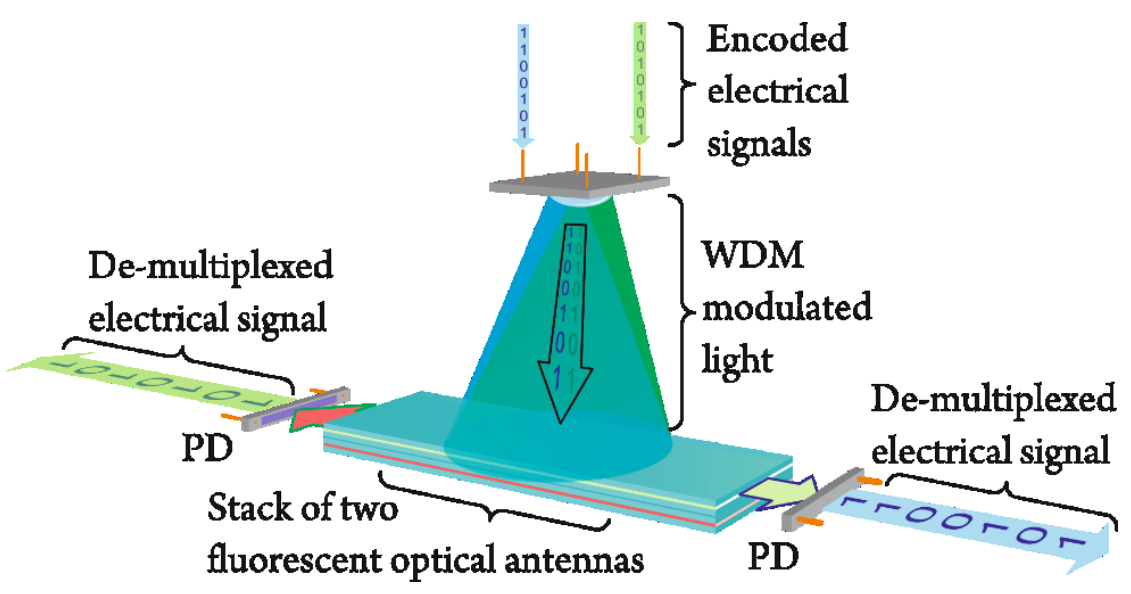

Figure 2. Schematic representation of the WDM fluorescent antenna. The transmitted signal is multiplexed and drives blue and green light emitting diodes (LED). The receiver consists of a layer of Coumarin 6 attached to a photodiode (PD) and a layer of DCM attached to another PD. The blue light is primarily absorbed by the Coumarin 6 layer and the green light by the DCM layer. The resulting fluorescence is guided to the edges of the device, where it is detected by the two PDs. Each of the PDs is attached to a different layer and therefore primarily receives the signal encoded into the blue or green wavelength channel. Due to the geometry of the antenna, the SNR is enhanced, while the angular response of the detector is proportional to the cosine of the angle of incidence, giving a wide field-of-view. 

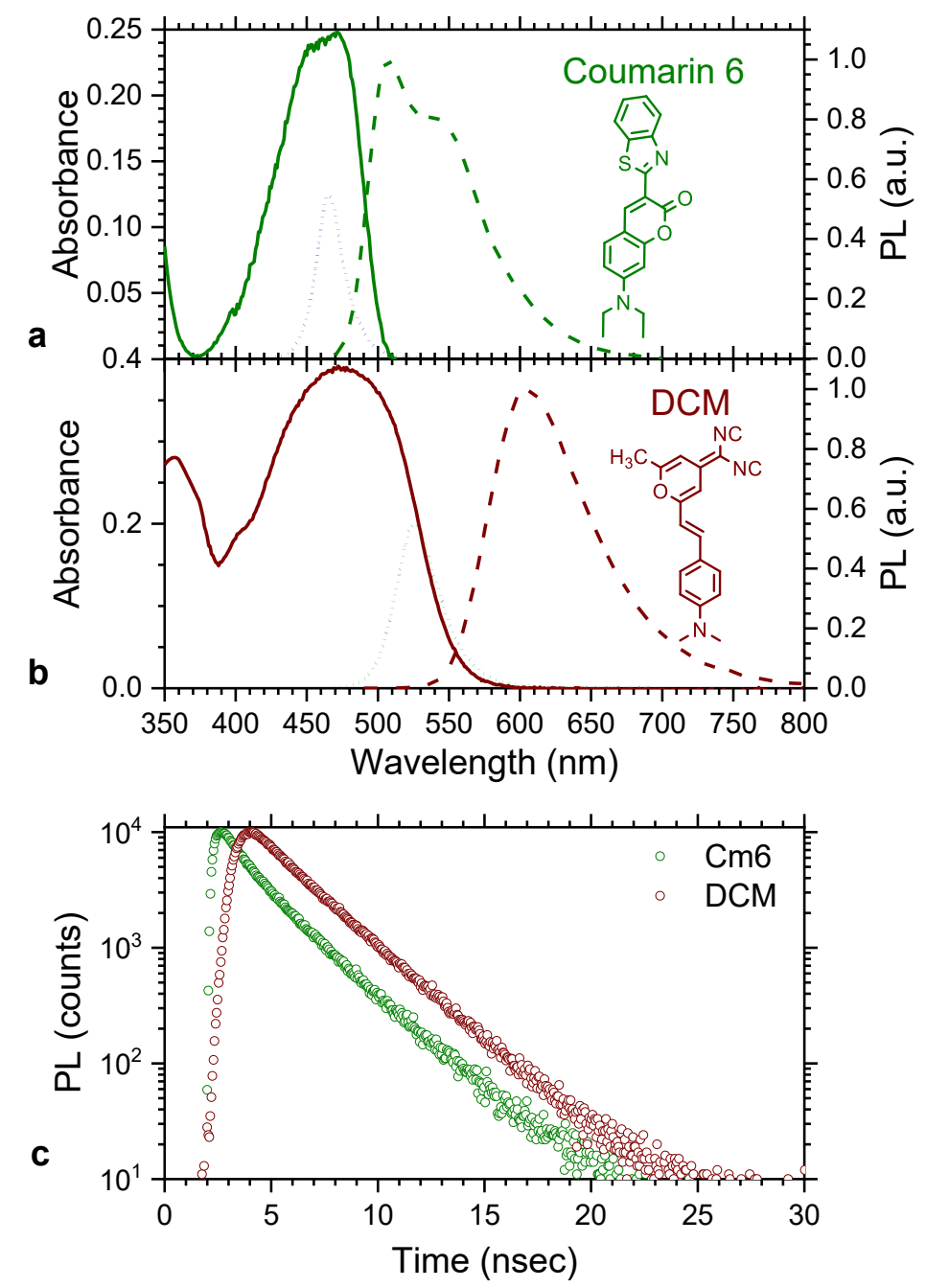

Figure 3. Photophysics of Cm6 and DCM in NOA68 epoxy. a) Absorbance and fluorescence (excited at $480 \mathrm{~nm}$ ) spectra of Cm6. The dotted line shows the electroluminescence spectrum of the blue LED, and the inset shows the molecular structure of Cm6. b) Absorbance and fluorescence (excited at $480 \mathrm{~nm}$ ) spectra of DCM. The dotted line shows the electroluminescence spectrum of the green LED, and the inset shows the molecular structure of DCM. c) Time resolved fluorescence measurement of the two dyes, excited at $375 \mathrm{~nm}$. The black line shows the instrument response function. 


\section{WILEY-VCH}

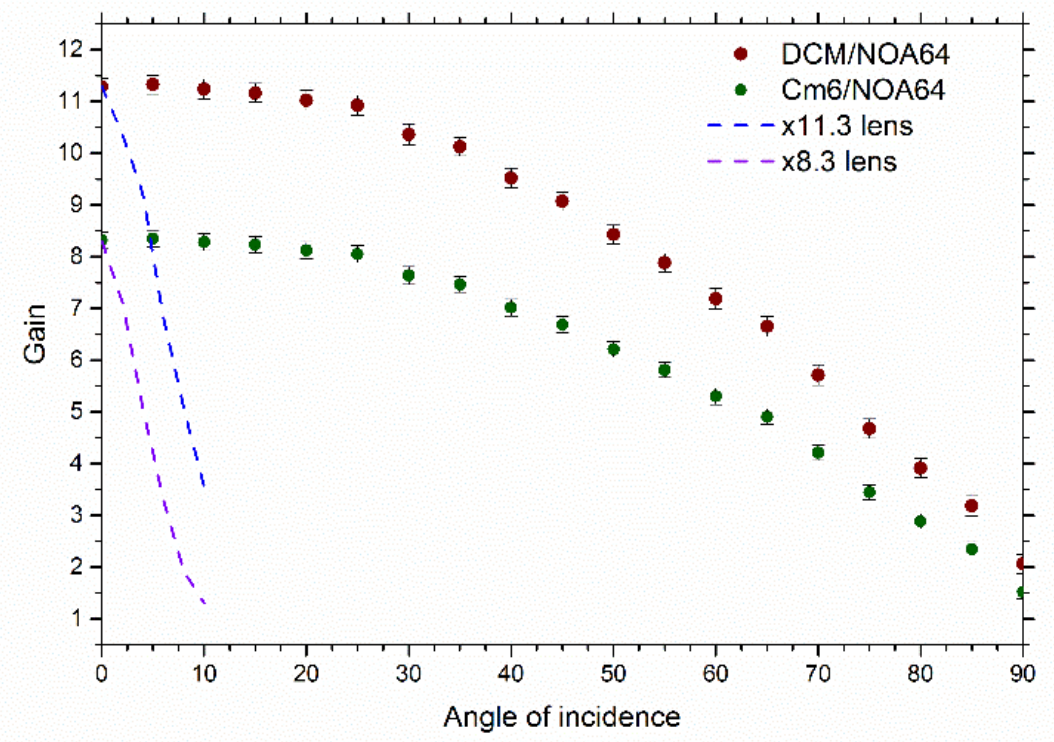

Figure 4. The gain of Cm6 in NOA68 epoxy, for the blue LED, and DCM in NOA68 epoxy, for the green LED, as a function of incident angle. The dashed lines correspond to gain measurements with a condenser lens $(\varnothing 50.8 \mathrm{~mm}, \mathrm{f}=32.0 \mathrm{~mm})$. 


\section{WILEY-VCH}

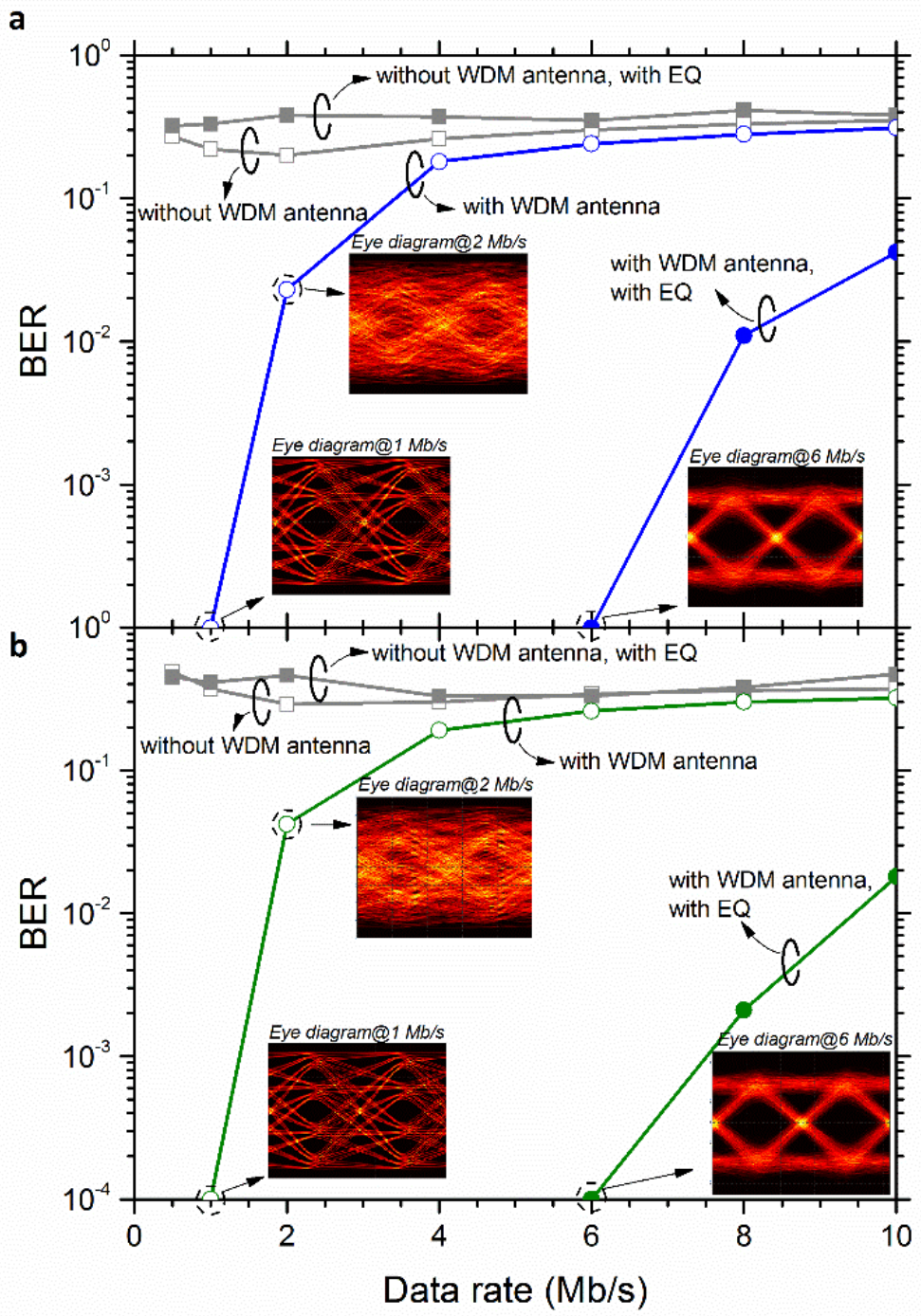

Figure 5. BER versus data-rate. a) blue LED channel performance, and b) green LED channel performance. Insets are eye-diagrams for each indicated data rate. 


\section{WILEY-VCH}

Copyright WILEY-VCH Verlag GmbH \& Co. KGaA, 69469 Weinheim, Germany, 2018.

\section{Supporting Information}

\section{Optical Antennas for Wavelength Division Multiplexing in Visible Light Communications beyond the Étendue limit}

Pavlos P. Manousiadis, Hyunchae Chun, Sujan Rajbhandari, Dimali A. Vithanage, Rahmat Mulyawan, Grahame Faulkner, Harald Haas, Dominic C. O'Brien, Steve Collins, Graham A. Turnbull*, and Ifor D.W. Samuel*

\section{DEVICE FABRICATION}

Fluorescent antenna fabrication. Figure S1 depict the details of the fabrication process. The dyes Coumarin 6 (Cm6) and 4-(Dicyanomethylene)-2-methyl-6-(4-dimethylaminostyryl)-4Hpyran (DCM), where chosen for de-multiplexing the blue and green components of the light signal, respectively. The two components of the device were fabricated using the same method. Each dye was separately mixed with the UV curable epoxy, Norland 68 (NOA68). For each solution, a concentration of $4 \mathrm{mg} / \mathrm{ml}$ was used. The solutions were prepared by weighing the appropriate mass of dye then adding the corresponding volume of epoxy. The solution was mixed, with a magnetic stirring bar for 24 hours. The ends of two microscope slides were covered in black tape that was trimmed to the same thickness as the microscope slide using a scalpel. A volume of ca. $0.25 \mathrm{ml}$ of dye/epoxy solution was spread on the slide which was placed between four spacers on a flat steel plate (see fig. S1). The spacers were made from aluminium, in a disk shape and their thickness was chosen to be the thickness of the two microscope slides plus $100 \mu \mathrm{m}$. A second microscope slide was placed on top of the first slide. These were pressed together under a uniform pressure from a $3 \mathrm{~mm}$ thick fused silica plate so that the thickness of the antenna was defined by the spacer thickness. A flood UV exposure, using an $8 \mathrm{~W}$ UV lamp at $365 \mathrm{~nm}$, for three minutes followed to cure the epoxy. 


\section{WILEY-VCH}
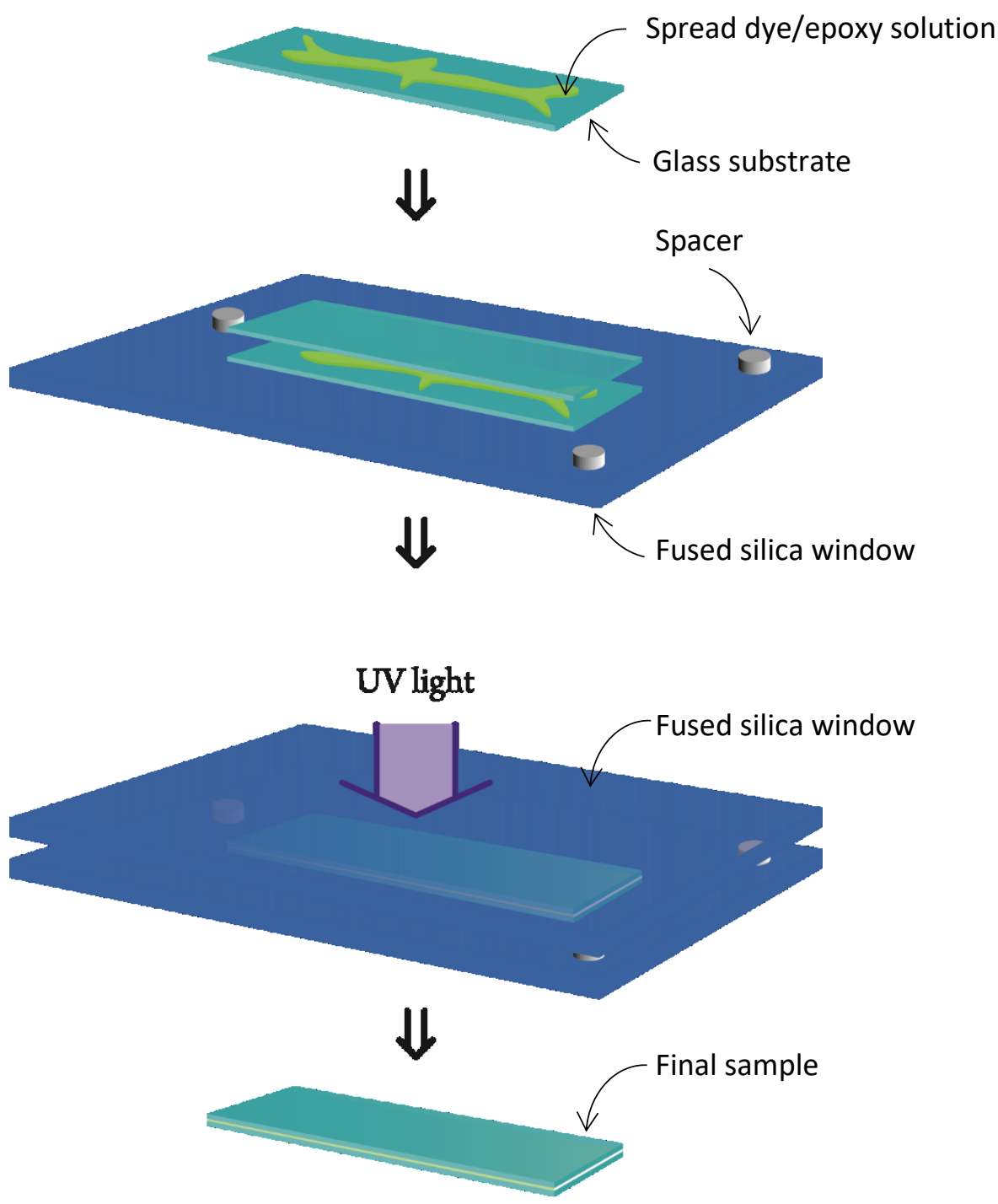

Figure S1. Schematic representation of fluorescent antenna fabrication process. From topdown: initially, a small volume of dye/epoxy solution is spread on a microscope slide (see top part of the figure above), a second slide is places on top, the slide/solution/slide layer is pressed between two fused silica windows where the space between them is defined by spacers, and the final stage is an UV curing of the solution.

WDM fluorescent antenna fabrication. The two components, of the WDM fluorescent antenna, were attached to each other by insulating tape (see fig. S2). Between the two components a small gap was formed $(\sim 0.1 \mathrm{~mm})$ by using two pieces of tape as spacers. 


\section{WILEY-VCH}

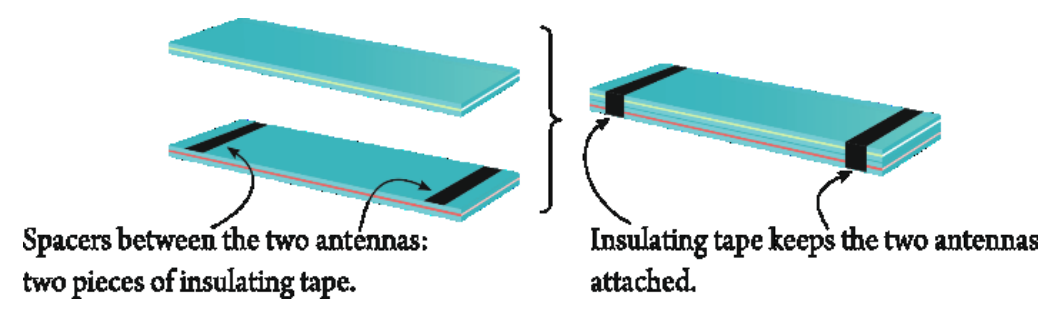

Figure S2. Schematic representation of how the WDM antenna was made. Two fluorescent antennas were held together by insulating tape, an $d$ insulating tape was also used as spacer between them. Finally, the two components are attached by an insulating tape.

\section{DEVICE ABSORBANCE}

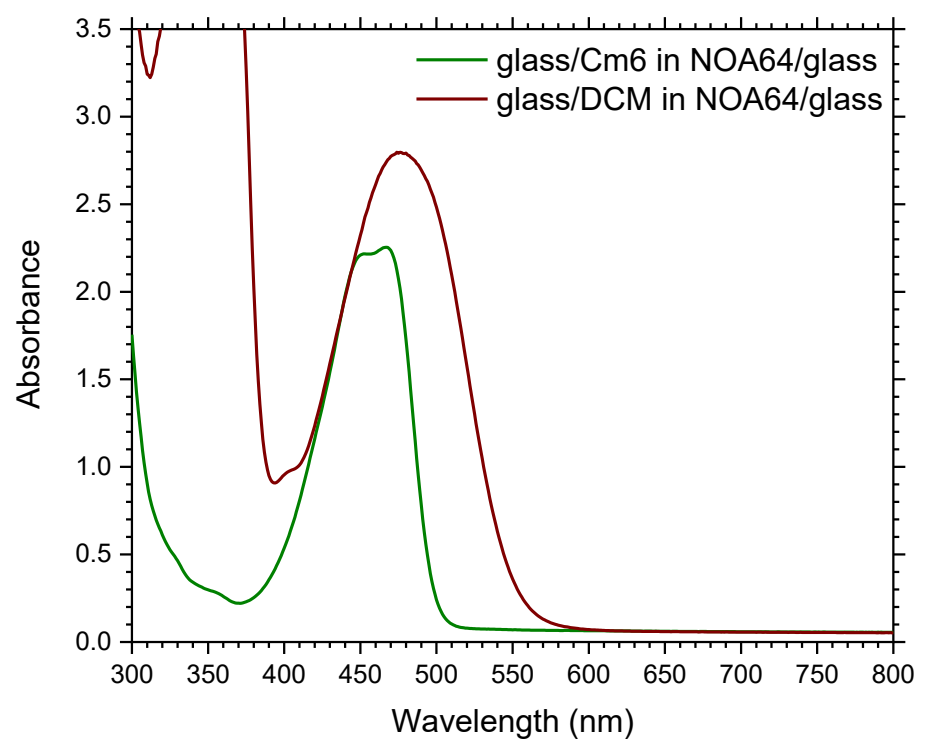

Figure S3. Absorbance of the different parts of the final device. 